\title{
CrystEngComm
}

CrossMark $<$ click for updates

Cite this: CrystEngComm, 2015, 17, 261

Received 19th August 2014, Accepted 30th October 2014

DOI: 10.1039/c4ce01711k

www.rsc.org/crystengcomm

\section{Simulation and modelling of MOFs for hydrogen storage}

\begin{abstract}
Yasemin Basdogan ${ }^{a}$ and Seda Keskin ${ }^{\star a b}$
Metal organic frameworks (MOFs) have received significant attention in recent years both from academia and industry since this new class of nanoporous materials has many potential advantages over traditional nanoporous materials in gas storage and separation applications. Hydrogen storage has been one of the most widely investigated applications of MOFs and recent experimental studies have shown that several MOFs are promising for hydrogen storage at low temperatures and moderate pressures. It is not practical to test every single MOF in the laboratory for hydrogen storage using experimental methods due to the very large number of existing MOF materials in the literature. Efforts to estimate the hydrogen storage performance of MOFs using molecular simulations and theoretical modelling play a very important role in identifying the most promising materials prior to extensive experiments. We review the current state of the art in molecular simulations and modelling of MOFs for hydrogen storage, compare experimental measurements and simulation predictions for hydrogen uptake of widely studied MOFs, discuss the main reasons for the discrepancy between experiments and simulations, and address the importance of developing theoretical models to predict the hydrogen storage performance of MOFs based on structural properties of materials prior to computationally demanding molecular simulations.
\end{abstract}

\section{Introduction}

The demand for alternative fuels is greater now than perhaps ever before because of the concerns over energy security, air quality, global warming and climate change. Among various renewable energy sources, hydrogen has received significant attention since it is abundant, environmentally friendly and offers higher power density (33.3 $\left.\mathrm{kWh} \mathrm{kg}^{-1}\right)$ than methane (13.9 $\mathrm{kWh} \mathrm{kg}^{-1}$ ) and gasoline (12.4 $\left.\mathrm{kWh} \mathrm{kg}^{-1}\right) .{ }^{1}$ A key challenge in preventing the hydrogen-driven energy future is the lack of an effective, economical and safe on-board hydrogen storage system. Current on-board hydrogen storage approaches include compressed hydrogen gas tanks, liquid hydrogen tanks, cryogenic compressed hydrogen, metal hydrides, nanoporous adsorbents and chemical hydrogen storage materials. ${ }^{2}$ High-pressure storage (5000-10 000 psi tank pressure) requires high capital cost and brings safety problems. Storage of hydrogen as a liquid requires cryogenic temperatures due to the boiling point of hydrogen $\left(-252.8^{\circ} \mathrm{C}\right.$ at atmospheric pressure), which is again costly and not safe. An alternative solution is to use nanoporous materials to store hydrogen at similarly

\footnotetext{
${ }^{a}$ Department of Chemical and Biological Engineering, Koc University, Rumelifeneri Yolu, Sariyer, 34450, Istanbul, Turkey. E-mail: skeskin@ku.edu.tr; Tel: +902123381362

${ }^{b}$ KÜTEM (Koc University TUPRAS Energy Center), Koc University, Rumelifeneri Yolu, Sariyer, 34450, Istanbul, Turkey
}

high density but substantially lower pressure. Due to the high interest in hydrogen storage, in 2003 the U.S. Department of Energy (DOE) initiated a research program with ambitious hydrogen storage targets of $6 \mathrm{wt} \%\left(45 \mathrm{~g} \mathrm{~L}^{-1}\right)$ for 2010 and $9 \mathrm{wt} \%$ (81 $\mathrm{g} \mathrm{L}^{-1}$ ) for 2015 at near-ambient temperature. Several different nanoporous materials such as zeolites, activated carbons, and carbon nanotubes have been proposed and widely investigated for hydrogen storage; ${ }^{3}$ however, there have been no materials that can reach the target values. Therefore, the DOE revised the targets in 2009 to be $4.5 \mathrm{wt} \%\left(28 \mathrm{~g} \mathrm{~L}^{-1}\right)$ for 2010 and $5.5 \mathrm{wt} \%\left(40 \mathrm{~g} \mathrm{~L}^{-1}\right)$ for $2015 .^{2}$ Design and development of new nanoporous materials that can achieve these new targets and store hydrogen efficiently and economically become crucial for a hydrogen energy-driven future.

A new group of nanoporous materials named metal organic frameworks (MOFs) has been recently identified to be promising for hydrogen storage. MOFs are composed of metal ligand complexes forming vertices of a framework that is connected by organic linkers. MOFs are synthesized from solvothermal reactions with metal and organic building blocks in organic solvents and several comprehensive studies are available about the synthesis methods of MOFs. ${ }^{4-20}$ MOFs typically have low densities $\left(0.2-1 \mathrm{~g} \mathrm{~cm}^{-3}\right)$, high surface areas (500-6500 $\mathrm{m}^{2} \mathrm{~g}^{-1}$ ), high porosities, and good thermal and mechanical stabilities. ${ }^{21}$ This combination of properties has made MOFs interesting materials for a wide range of potential applications, including gas storage, gas separation, catalysis, 
and biomedical, optical and electronic applications. ${ }^{22-27}$ The most important advantage of MOFs over other traditional nanoporous materials is the ability to tune the physical and chemical properties of the materials during synthesis. ${ }^{22,28}$ Theoretically, it is possible to synthesize an infinite number of MOFs by combining different metal ions with various organic linkers to obtain a large variety of materials.

MOFs exhibit excellent potential in gas storage due to their wide range of pore sizes, permanent pores in nanodimensions, chemical functionalities, low crystal densities, and good thermal and mechanical stabilities. ${ }^{8,9,29}$ Storage of hydrogen, carbon dioxide and methane in MOFs has been widely studied in the literature. ${ }^{30-32}$ Hydrogen storage has been the most commonly examined gas storage topic in the MOF literature both experimentally and theoretically. ${ }^{33}$ There is a very large literature on experimental hydrogen adsorption in MOFs and several reviews ${ }^{4,20,34}$ summarized the recent strategies to improve hydrogen uptake of MOFs such as impregnation, catenation, incorporation of open metal sites in MOFs, functionalization of organic linkers and hydrogen spillover methods.

Fig. 1(a) shows the rapid growth of research on hydrogen storage applications of MOFs in the past decade. Most of the experiments have focused on measuring hydrogen uptake of only one MOF or a few MOFs at a time. Considering the large number of available MOFs in the literature and challenges related to synthesizing new materials, it is not practical to test the hydrogen storage capacity of every single MOF using experimental methods. At this point, molecular simulations and modelling studies offer a great advantage to rapidly screen the large number of materials and identify the most promising ones for hydrogen storage applications. Efforts, resources and time can then be directed to these promising materials and more extensive studies can be carried out.

(a)

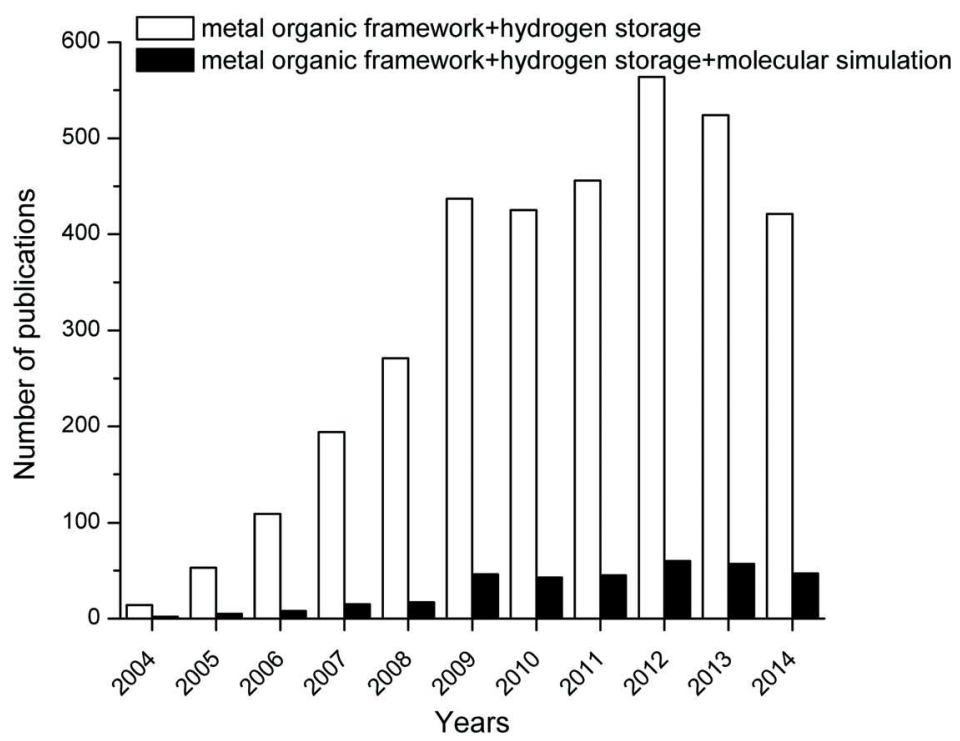

(b)
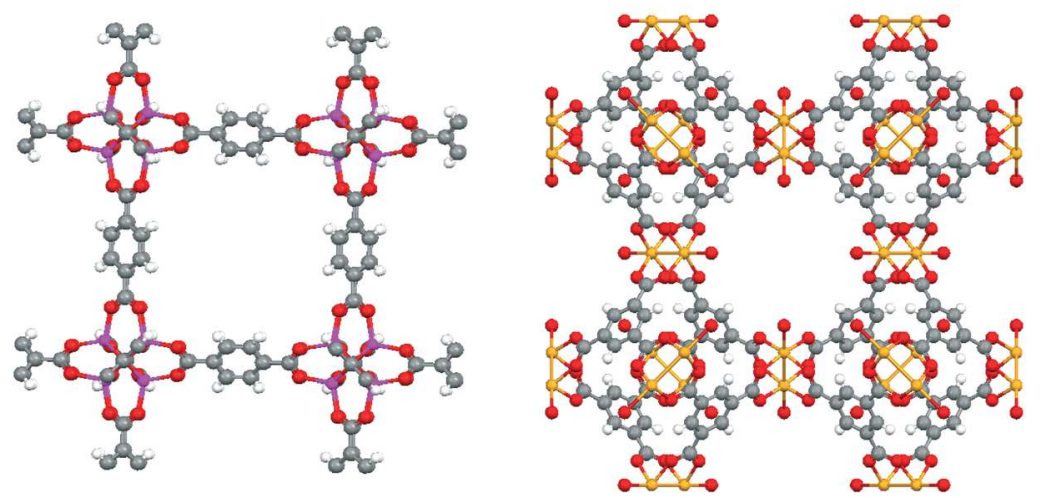

Fig. 1 (a) Open bars represent the number of publications featuring the terms 'metal organic framework' and 'hydrogen storage'. Closed bars represent the number of publications featuring the terms 'metal organic framework', 'hydrogen storage' and 'molecular simulation'. (Source: ISI Web of Science, retrieved October 20, 2014). (b) Unit cell crystal structure of IRMOF-1 viewed along the [100] direction (left) Zn: violet, O: red, C: gray and $\mathrm{H}$ : white. Unit cell crystal structure of CUBTC viewed along the [100] direction (right) Cu: orange, O: red, C: gray and H: white. 
As Fig. 1(a) suggests, the idea of using molecular simulations and modelling to assess the hydrogen storage potential of MOFs is a relatively new and rapidly growing area.

Although there are several excellent reviews on experimental hydrogen storage in MOFs as we will discuss on the following pages, there is no comprehensive review on molecular simulations and modeling of MOFs for hydrogen storage. Molecular simulation studies of MOFs are generally very briefly mentioned as a subsection in the current review papers. Simulations and modeling studies can offer significant information about the potential of MOFs for hydrogen storage prior to extensive experimental efforts, guide the experiments to promising materials by screening a large number of MOFs and help to design new materials by providing molecular-level knowledge. In this review, we focus on opportunities and challenges related to using molecular simulations and developing theoretical models for hydrogen storage in MOFs. We discuss the most commonly used molecular simulation method, Grand Canonical Monte Carlo (GCMC), for assessing hydrogen adsorption in MOFs. In addition to GCMC simulations which are used to predict the hydrogen uptake capacities of MOFs, quantum-level calculations are also performed in the literature to compute the binding energies of hydrogen to MOFs. We did not include quantum-level $a b$ initio studies of hydrogen adsorption in MOFs in this review since comprehensive reviews already exist on this topic. ${ }^{20,32}$ We compare the results of experimental measurements and simulation predictions for hydrogen uptake of widely studied MOFs and discuss the main reasons for the discrepancy between experiments and simulations. We then address theoretical models that can predict the hydrogen uptake of MOFs based on structural properties of the materials without performing molecular simulations and/or experiments.

\section{Molecular simulations for hydrogen adsorption in MOFs}

Grand Canonical Monte Carlo (GCMC) simulations have been used to calculate the hydrogen uptake of MOFs. GCMC is a widely used simulation technique that mimics the experimental measurements to calculate the adsorption isotherms of gases in MOFs. ${ }^{35}$ In an experimental set-up, the adsorbed gas is in equilibrium with the gas in the reservoir and the equilibrium conditions are that the temperature and chemical potential of the gas inside and outside of the adsorbent must be equal. In the grand canonical ensemble, the temperature, volume and chemical potential are fixed and the number of particles is allowed to fluctuate during the simulation at an imposed temperature and chemical potential. The GCMC simulations consist of millions of random molecule insertions, deletions, translations and rotations based on the Boltzmann-type weighting principle. Observations of GCMC simulations are directly comparable with the output of adsorption experiments. GCMC simulations calculate the absolute amount of adsorbed gas in a material's pores, whereas experiments measure the excess adsorption amount.
Therefore, results of GCMC simulations are converted to excess adsorption in order to make a comparison with experiments. The excess adsorption is determined from the absolute adsorption by subtracting the pore volume of the MOF, which can be calculated as suggested in the literature, ${ }^{36}$ multiplied by the density of the gas phase, which can be calculated by an equation of state. ${ }^{30}$ The excess adsorption can be decreased in the high-pressure region, while the total gas uptake should increase monotonically with an increase in the pressure because of the contribution from the large bulk density of the adsorbate. ${ }^{37}$ The difference between absolute and excess adsorption is important in interpretation of adsorption data. For example, GCMC simulation results on two most widely studied MOFs, IRMOF-1 (isoreticular metal organic framework-1) and CuBTC (copper benzenetricarboxylate), illustrated in Fig. 1(b), showed that IRMOF-1 adsorbs more hydrogen than CuBTC based on absolute adsorption; however, CuBTC shows a larger excess adsorption than IRMOF-1. ${ }^{38}$ This is due to the larger free volume of IRMOF-1 compared to that of CuBTC. Therefore, in comparing the hydrogen uptake of different MOFs, considering the hydrogen density within the pore volume of the MOF is important.

We summarized the details of GCMC simulations which have been performed to compare hydrogen adsorption in MOFs with the experimental measurements in Table 1. Most of these studies have focused on a few specific MOF groups such as IRMOFs and COFs (covalent organic frameworks, in which the organic building units are held together by strong covalent bonds rather than metal ions). The accuracy of a GCMC simulation depends on the accuracy of the force fields that describe the interatomic interactions between hydrogen molecules and MOF atoms. The potential models used in molecular simulations must be first validated against the experimental data to show the reliability of the simulations. Table 1 shows that generic force fields, universal force field (UFF), ${ }^{39}$ DREIDING $^{40}$ and OPLS (optimized potential for liquid simulations $)^{41}$ have been used in hydrogen adsorption simulations. These force fields have been developed to predict structures and dynamics of organic and main group inorganic molecules and therefore they roughly predict binding energies of non-bonded interactions between MOFs and hydrogen molecules. In most studies, UFF is used to predict the hydrogen adsorption isotherm of a particular MOF and compared with the available experimental data. If the agreement between simulations and experiments is not good, another force field such as DREIDING or OPLS is tried to obtain a better agreement. The potential parameters for metal atoms of MOFs are taken from the UFF even if the DREIDING force field is used for non-metal atoms of the framework since these parameters are not available from DREIDING. As can be seen from Table 1, only a small number of studies has carried out quantum mechanical calculations to develop new force fields for specific MOF-hydrogen interactions. In these studies, a multi-scale simulation method that combines the quantumlevel calculations and GCMC simulations has been used to predict hydrogen uptake capacities of MOFs. The interaction 
Table 1 GCMC simulations for hydrogen uptake of MOFs

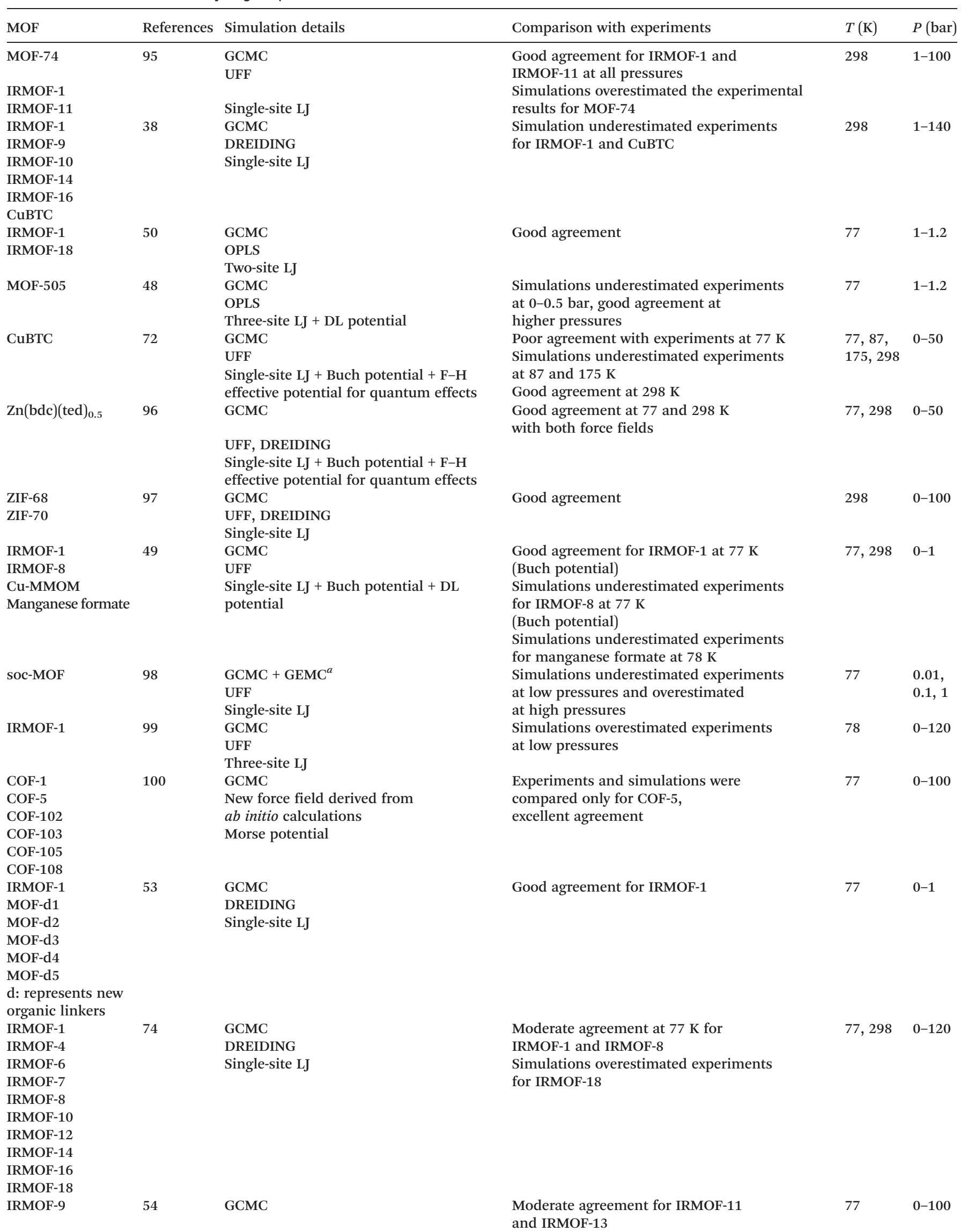


Table 1 (continued)

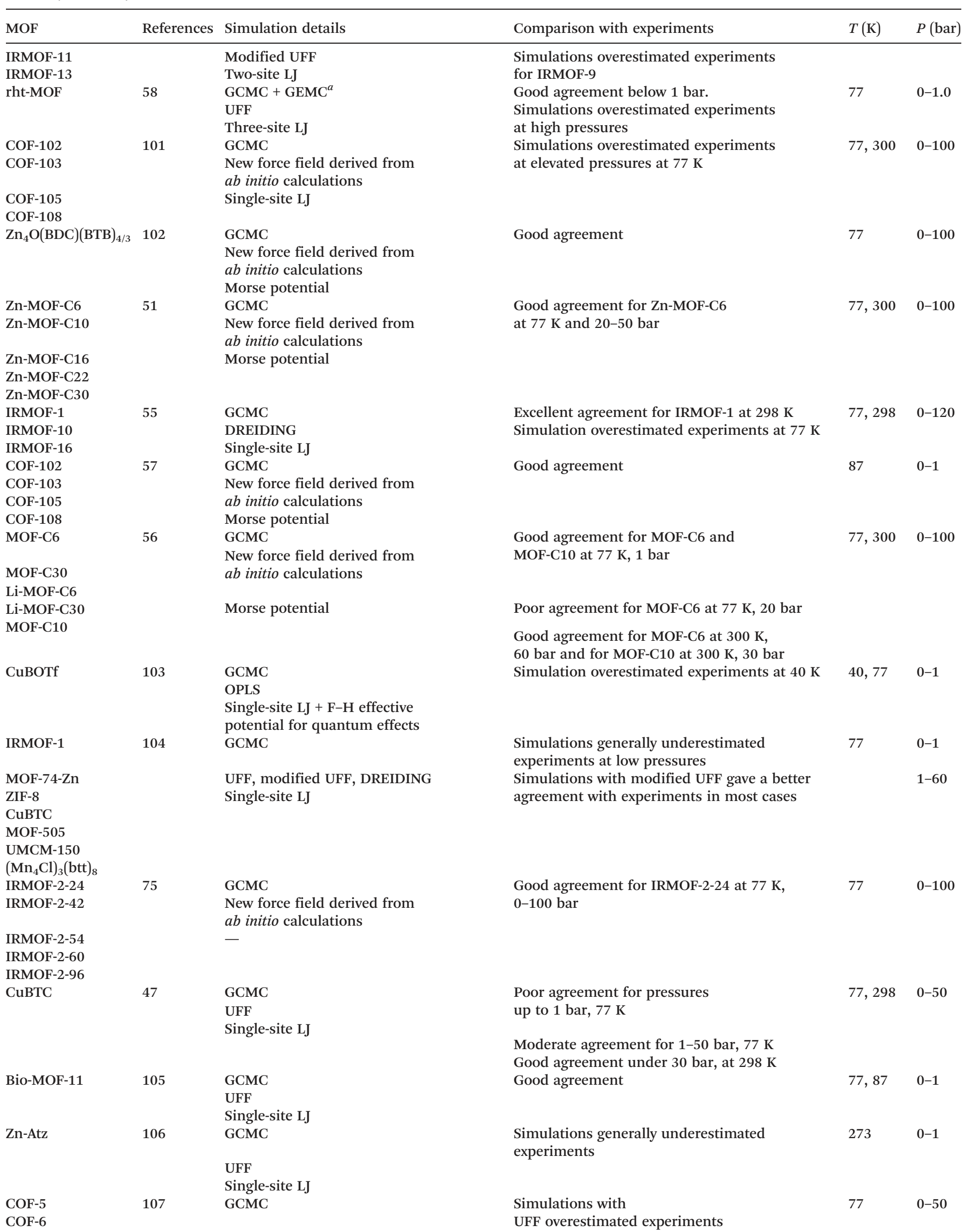


Table 1 (continued)

\begin{tabular}{|c|c|c|c|c|c|}
\hline COF-10 & & $\begin{array}{l}\text { UFF + DREIDING } \\
\text { Single-site LJ }\end{array}$ & & & \\
\hline & & & $\begin{array}{l}\text { Good agreement between simulations } \\
\text { with DREIDING and experiments }\end{array}$ & & \\
\hline Cu-TDPAT & 108 & $\begin{array}{l}\text { GCMC } \\
\text { UFF + DREIDING }\end{array}$ & $\begin{array}{l}\text { Good agreement between simulations } \\
\text { with DREIDING and experiments }\end{array}$ & 77,298 & $0-60$ \\
\hline
\end{tabular}

energies between hydrogen molecules and MOF atoms are obtained from quantum mechanical calculations and calculated interaction energies are then fitted to an appropriate potential function to be used as force fields in GCMC simulations. More details of quantum-level calculations ( $a b$ initio methods) and fitting the force field parameters can be found in the literature. ${ }^{30}$

In most of the molecular simulations, the Lennard-Jones $(\mathrm{LJ})^{42}$ potential is used to describe hydrogen-hydrogen interactions. The single-site LJ 12-6 model is the most commonly used, of which the Buch potential ${ }^{43}$ is known to reproduce the experimental bulk equation of state for hydrogen accurately. ${ }^{44}$ The two-site LJ model and the model of Darkrim and Levesque (DL) ${ }^{45}$ which is used to consider the quadrupole moment of hydrogen molecules have also been used in some simulation studies. The Morse potential model has been recently used more frequently for hydrogen simulations due to its adjustability in fitting force fields. ${ }^{30}$ At low temperatures and high densities, hydrogen exhibits quantum effects which should be taken into account in order to increase the accuracy of the simulations. Some studies have used Feynman-Hibbs $(\mathrm{FH})^{46}$ effective potential to account for quantum effects in adsorption simulations below $100 \mathrm{~K}$. The bulk equation of state for hydrogen was calculated at $77 \mathrm{~K}$ from the GCMC simulations using the classical Buch potential, the $\mathrm{FH}$ effective Buch potential and the DL model with $\mathrm{FH}$ corrections, and results of different potentials are compared with the experimental data. ${ }^{47}$ The equation of state for hydrogen was accurately reproduced by the $\mathrm{FH}$ effective Buch potential, whereas the FH effective DL potential slightly overestimated the density. The Buch potential has been shown to accurately reproduce the fluid properties of hydrogen over a very wide range of temperatures and densities. ${ }^{48}$ Johnson's group $^{49}$ examined the importance of framework charges on hydrogen adsorption as a function of pressure and temperature. They tested the effect of framework charge interactions with the hydrogen quadrupole using the DL potential and computing charges of IRMOF-1 from $a b$ initio calculations. They showed that charge-quadrupole interactions enhance hydrogen adsorption of IRMOF- 1 at $77 \mathrm{~K}$ and pressures less than 5 bar. However, the effect of charge-quadrupole interactions is much less significant at higher pressures and almost not important at room temperature. It is important to note that the effect of the electrostatic potentials, both solid-fluid (MOF-hydrogen) and fluid-fluid (hydrogen-hydrogen), is always to increase the number of adsorbed molecules. The contribution of the solid-fluid electrostatics to the adsorption was found to be maximum at zero pressure. The solid-fluid contribution to adsorption decreases monotonically, contributing a few percent at pressures above 25 bar. Overall, the presence of electrostatic interactions results in an amount adsorbed that is about $40-45 \%$ higher than that given by the LJ core alone for low pressures. ${ }^{49}$

Table 1 shows that there is generally a good agreement between simulation results and experimental uptake measurements, suggesting that molecular simulations can be used to get initial estimates about the hydrogen storage capacity of MOFs. Most of the simulation studies listed in Table 1 concluded that the hydrogen storage capacity of MOFs is similar to that of carbon nanotubes and higher than that of zeolites at $77 \mathrm{~K}$. The GCMC simulations listed in Table 1 were generally performed at $77 \mathrm{~K}$ and/or $298 \mathrm{~K}$ and at low pressures to represent the desired operating conditions for hydrogen storage. Several MOFs were reported to reach the gravimetric DOE targets at $77 \mathrm{~K}$ but not at room temperature, and hence, practical usage of MOFs must be further investigated at near-ambient temperatures. At that point it is important to note that the gravimetric target of the DOE refers to the entire storage system including all hardware such as tank, valves, and heating/cooling systems. Therefore the actual storage capacity must be even higher to use MOFs for hydrogen-powered vehicles.

Molecular simulation studies listed in Table 1 do not only examine the hydrogen uptake of MOFs but also provide molecular-level information about hydrogen-MOF interactions and strategies to enhance hydrogen storage capacity of MOFs. For example, GCMC simulations of Yang and Zhong ${ }^{50}$ showed that metal clusters are the preferential adsorption sites for hydrogen molecules and the effect of organic linkers becomes evident with increasing loadings, which has been important information for further design and development of new MOFs for hydrogen storage. They then combined quantum-level 
calculations with GCMC simulations and showed that open metal sites can provide a favorable impact on hydrogen adsorption in MOFs especially at low pressures, while at high pressure structural properties such as pore volume become more important. ${ }^{48}$ Han et al. ${ }^{51}$ suggested designing MOFs with larger organic linkers (increased aromatic content) in order to enhance hydrogen adsorption at high pressures since their simulations demonstrated that the dominant adsorption site at high pressure is the organic linker. Getman et al. ${ }^{52}$ combined GCMC simulations with $a b$ initio calculations to explore metal alkoxide functionalization for improving hydrogen storage in MOFs, IRMOF-1, IRMOF-10, IRMOF-16, UiO-68 and UMCM-150. They showed that lithium and magnesium alkoxides physically bind hydrogen, and manganese, nickel, and copper alkoxides chemically bind hydrogen. Magnesium alkoxide was identified as a promising functional group for enhanced ambient-temperature hydrogen storage in all MOFs they studied.

Zhang et $a l .{ }^{53}$ developed an effective method named computer tomography for materials ( $\mathrm{MCT}$ ) to exhibit all the preferential adsorption sites of hydrogen in a MOF material. In this method, during GCMC simulations, all configurations of hydrogen molecules were recorded at certain intervals and the overall-averaged distribution of hydrogen molecules adsorbed in three-dimensional space was calculated statistically on the basis of all of these configurations. After testing this method on IRMOF-1 and getting information on how hydrogen molecules interact with MOF atoms, they designed new MOFs that exhibit better hydrogen storage capacity than IRMOF-1. Jung et al. ${ }^{54}$ performed GCMC simulations to understand the effect of catenation, where two or more identical frameworks are intergrown at the expense of the pore volume, on hydrogen adsorption and identified the adsorption sites of IRMOFs. They concluded that small pores generated by catenation can confine the hydrogen molecules more densely and therefore the storage capacity of catenated IRMOFs could be higher than that of non-catenated counterparts. Ryan et al. ${ }^{55}$ examined the same question, benefits of catenation on hydrogen adsorption, and demonstrated that catenation is a useful strategy to enhance hydrogen adsorption in MOFs at low temperatures and pressures but not at ambient temperature. At high loadings, catenated MOFs have less free volume than their non-catenated versions, and smaller free volume limits the hydrogen uptake both on gravimetric and volumetric basis. Molecular simulations by Han et al. ${ }^{56}$ and Cao et al. ${ }^{57}$ showed that Li-doped MOFs and COFs can store more hydrogen than non-doped ones under the same conditions since hydrogen strongly binds to the positively charged Li cations doped on MOFs. Similarly, simulations by Babarao et al. ${ }^{58}$ suggested that ionic MOFs can enhance hydrogen adsorption at low loadings due to the strong interactions between hydrogen molecules and extra-framework ions of the MOF.

The most important contribution of molecular simulations is perhaps design of new materials that can achieve high-performance hydrogen storage prior to experiments. Snurr's group ${ }^{59}$ computationally designed and characterized a MOF named NU-100 and predicted its hydrogen storage capacity using molecular simulations. On the basis of promising results obtained from simulations, they experimentally synthesized this MOF and showed the good agreement between experiments and simulations for high surface area. NU-100 exhibited high hydrogen storage $\left(164 \mathrm{mg} \mathrm{g}^{-1}\right)$ at 70 bar and $77 \mathrm{~K}$ which agrees well with the predictions of simulations. The hydrogen storage of NU-100 at low pressure and $77 \mathrm{~K}\left(99.5 \mathrm{mg} \mathrm{g}^{-1}\right)$ is one of the highest values reported to date among various MOFs. Here, it is important to note that throughout this review, we avoid giving extensive quantitative information about the hydrogen uptake capacity (wt\%) of all MOFs because there are several reviews in the literature that list the uptake values. For example, the uptake capacity of MOFs that show high hydrogen storage at $77 \mathrm{~K}$ was listed by Broom et al. ${ }^{60}$ (COF-102, $6.86 \mathrm{wt} \%, 20 \mathrm{bar}$ ), simulated hydrogen uptake capacity of several MOFs at various pressures and temperatures was listed by Jiang et al. ${ }^{31}$ (IRMOF-8, $11.1 \mathrm{wt} \%$, 100 bar, $77 \mathrm{~K}$ ), experimental hydrogen uptake capacity of highly promising MOFs at $77 \mathrm{~K}$ was listed by Xiang et al. ${ }^{30}$ (MOF-177, 7.5 wt\%, 70 bar) where data in parentheses represent materials with the best uptake performance. In addition to these, Sculley et al. ${ }^{61}$ provided experimental hydrogen uptake capacity (both wt $\%$ and $\mathrm{g} \mathrm{L}^{-1}$ ) of $>135$ MOFs at 77 and $298 \mathrm{~K}$ under various pressures (NU-100, $9.95 \mathrm{wt} \%$, 56 bar, $77 \mathrm{~K}$ ), and Suh et al. ${ }^{4}$ listed experimental hydrogen uptake capacity (both wt\% and $\mathrm{g} \mathrm{L}^{-1}$ ) of $>110$ MOFs without open metal sites (MOF-210, $8.6 \mathrm{wt} \%, 80 \mathrm{bar}, 77 \mathrm{~K}$ and) and $>90$ MOFs with open metal sites (NU-100 same as reported above) at different temperatures and pressures.

As shown in Table 1, most of the molecular simulation studies for hydrogen storage have focused on a single MOF or a few MOFs having similar topology. In Fig. 2, we compared the results of GCMC simulation with the experiments for

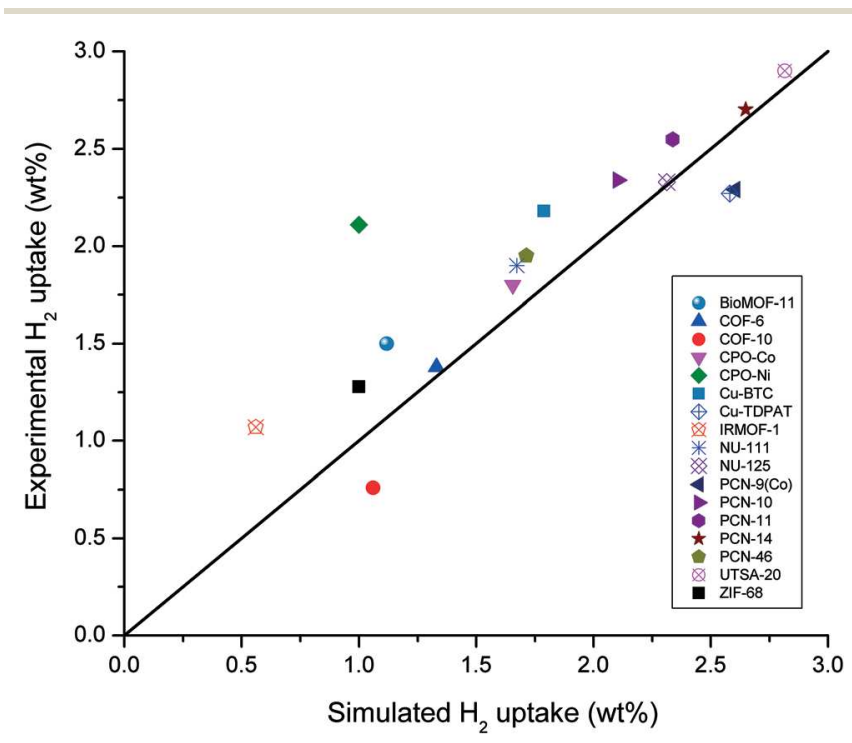

Fig. 2 Comparison of simulation results with experimental data for hydrogen uptake of widely studied MOFs at $77 \mathrm{~K}$ and 1 bar. 
hydrogen uptake of seventeen widely studied MOFs in the literature (BioMOF-11, COF-6, COF-10, CPO-Co, CPO-Ni, Cu-BTC, Cu-TDPAT, IRMOF-1, PCN-9-Co, PCN-10, PCN-11, PCN-14, PCN-46, UTSA-20, NU-125, NU-111, and ZIF-68, where CPO means coordination polymers of Oslo, UTSA means University of Texas at San Antonio, PCN means porous coordination network, and ZIF means zeolite imidazolate framework) at $77 \mathrm{~K}$ and 1 bar. These simulations were performed using a generic force field, UFF, and representing the hydrogen molecule as a single-site LJ sphere. Simulation results were converted to excess uptake in order to make an accurate comparison with the experimental data. One important outcome of Fig. 2 is that although these MOFs are the most widely studied materials in the literature, none of them is able to reach the desired hydrogen uptake targets. The MOF that shows the maximum hydrogen uptake in Fig. 2 is UTSA-20 with a capacity of $2.9 \mathrm{wt} \%$, which is still way below the target for 2015 .

Fig. 2 shows that there is a good agreement between the predictions of molecular simulations and experiments $\left(R^{2}=0.78\right)$ even though a generic force field was used and quantum effects were ignored in molecular simulations. This suggests that molecular simulations can be used to make accurate estimates about the hydrogen storage capacity of different types of MOFs. Simulations underestimated the hydrogen uptake of some MOFs such as bioMOF-11 and CPO-Ni and overestimated the uptake of some others such as COF-10 and ZIF-68. There are several reasons for the discrepancy between simulations and experiments such as assumptions used in simulations and problems related to experiments. We briefly discuss these assumptions below:

- The potential models and parameters used in the simulations have a significant effect on the final predictions of molecular simulations. Molecular simulations performed with a generic force field may give results that agree well with the experiments for a single MOF but deviate from the experimental measurements for another MOF. The large chemical variety of MOFs and the lack of proper force field parameters are some of the challenges in studying the hydrogen adsorption capacity of MOFs at the molecular level. Predictions of multi-scale simulations are generally better than the predictions of simulations based on generic force fields in most cases since hydrogen-MOF interactions are computed at the quantum level in the former. Furthermore, this type of quantum-level calculations can offer additional information about specific site-site interactions that may be useful in computational design of new materials. On the other hand, developing new force fields based on experimental data of a MOF and using this force field for a different group of MOFs may give misleading results. It is better to develop new force fields using ab initio calculations for a group of MOFs and then transfer the same force field for MOFs that have similar chemical and topological properties. Of course, developing high-accuracy force fields from quantum mechanical calculations is challenging and time-consuming. These calculations must be carefully performed using the most realistic models and the highest accuracy level of theory. ${ }^{32}$
- Another important assumption of molecular simulations is the rigid framework assumption. Almost all of the molecular simulations for hydrogen adsorption in MOFs used the rigid framework assumption because it saves a significant amount of computational time, and molecular simulations should be performed for multiple materials on time scales shorter than the same materials can be assessed experimentally. In rigid simulations, the framework atoms are fixed at their crystallographic positions which are usually obtained from $\mathrm{X}$-ray studies of experiments. There is no need to evaluate intra-framework interactions and the interactions between hydrogen molecules and MOF atoms can be pretabulated on a grid which significantly accelerates the simulations. Since adsorption involves low-energy equilibrium configurations, the flexibility of the framework has a marginal effect on the adsorption of gas molecules. ${ }^{62}$ However, several MOFs are reported to be flexible and undergo a structure transition in contact with the adsorbate molecules. ${ }^{63-67}$ GCMC simulations must be combined with the molecular dynamics simulations to represent the effects of framework transition of a flexible MOF on hydrogen uptake; however, this type of study is very limited in the literature. ${ }^{68,69}$ Pantatosaki et al. ${ }^{69}$ have recently used molecular dynamics to show that flexibility of the imidazolate linkers in ZIF-3 and ZIF-8 is of little importance for hydrogen adsorption but the framework mobility significantly influences the kinetics of hydrogen in both materials, particularly in ZIF-8, wherein hydrogen molecules experience tighter fitting as they pass through the narrow pores.

- Almost all of the molecular simulation studies apply boundary conditions to represent infinite MOF networks considering only intra-crystalline pores. However, in real synthesized MOFs inter-crystalline pores and/or defects may exist. The boundary effects of experimental samples are not considered by molecular simulations. In other words, molecular simulations assume defect-free perfect crystals in contrast to experiments. Simulation of MOFs by considering crystal defects would be an interesting study to understand the importance of boundary effects since this type of work has not been done yet.

- The most critical problem of the gas uptake experiments of MOFs is related to the purity of materials. MOFs are generally synthesized using hydrothermal or solvothermal techniques in which crystals slowly grow from a hot solution of metal precursor. ${ }^{6,9,15,70,71}$ The solvents used in the synthesis of MOFs remain in the pores of the framework and an activation procedure, generally heating, is required to remove the solvents from the structure to make the pores available for guest molecules. As-synthesized MOFs may have solvents trapped in their pores and these solvent molecules may significantly affect gas uptake measurements. For example, Liu et $a l .{ }^{72}$ provided a review of the literature for hydrogen adsorption in CuBTC and addressed that the preparation and activation process has an enormous impact on the adsorption capacity, surface area and pore volume of the material. Keskin et al. $^{73}$ compared experimental hydrogen adsorption isotherms of CuBTC at $77 \mathrm{~K}$ both at the low- and at the high- 


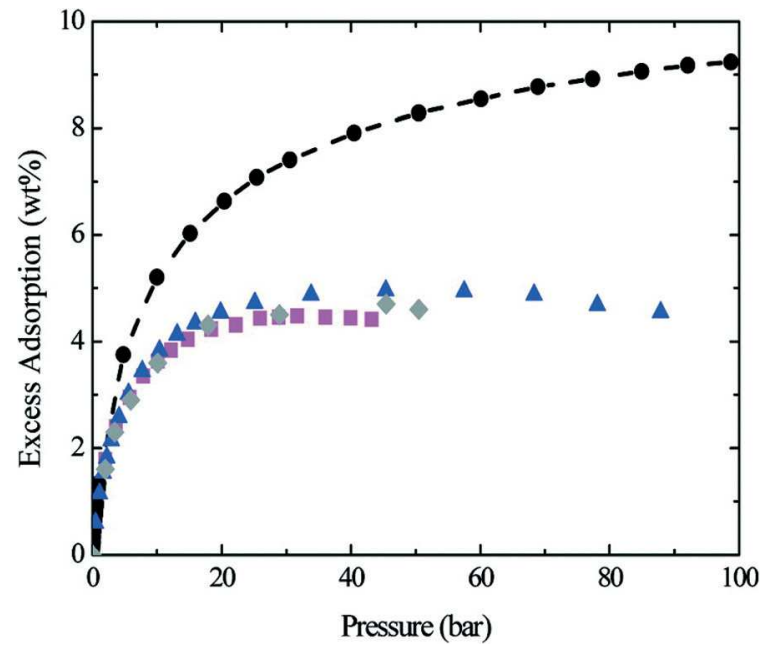

Fig. 3 Comparison of the predicted high-pressure $\mathrm{H}_{2}$ adsorption isotherm in IRMOF-1 by Yang and Zhong ${ }^{50}$ with experiments at $77 \mathrm{~K}$. Circles: simulations by Yang and Zhong, ${ }^{50}$ and the triangles, diamonds, and squares are experimental data from Wong-Foy et al., ${ }^{110}$ Panella et al., ${ }^{111}$ and Dailly et al., ${ }^{112}$ respectively. Reprinted with permission from ref. 73 . Copyright (2009) American Chemical Society.

pressure regime measured by different research groups and showed that these isotherms are different from each other due to the varying purity of the samples used by different research groups. It is important to remember that the influence of purity or integrity of a material is more pronounced for MOFs compared to other nanoporous materials such as zeolites because guest molecules can be removed from zeolites' pores by calcining at high temperatures, whereas some MOFs may not be stable at high temperatures. ${ }^{73}$

- Finally, comparing experiments and simulations over a wide range of operating conditions (pressures, temperatures) is a must. Most of the simulation studies compare their results with the experiments over a very narrow range of pressure ( $0-1$ bar) and then extend their simulations to high pressures, which may significantly deviate from experiments. Fig. 3 shows the comparison of simulated high-pressure hydrogen uptake of IRMOF-1 with experiments at $77 \mathrm{~K}$. The potential parameters for hydrogen-IRMOF-1 interactions were fitted using experimental data up to 1 bar and then the high-pressure hydrogen adsorption isotherm was predicted up to 100 bar based on these parameters..$^{50}$ As a result, simulations overpredicted the experimental measurements at the high-pressure regime, even though the low-pressure adsorption data were in excellent agreement with the experiments. Therefore, in developing interatomic potentials based on experimental data from a MOF, a broad range of physical conditions from multiple samples should be used to increase the reliability of the potential models.

\section{Theoretical models to predict hydrogen adsorption in MOFs}

An alternative approach to carrying out computationally demanding molecular simulations to predict the hydrogen uptake capacity of MOFs is developing theoretical models that can estimate the uptake capacity based on easily measurable or computable structural properties of MOFs such as pore volume, surface area, crystal density, etc. Developing this type of simple theoretical models does not require extensive calculations such as force field development. Furthermore, these models can be very helpful to learn which structural properties affect the hydrogen storage capacity of MOFs. Based on this learning, experimental studies can be guided to design new materials with the desired structural properties that will reach the storage targets.

The structural properties that affect the hydrogen storage of MOFs must be identified prior to development of these models. Frost $e t a l^{74}$ examined the effects of surface area, free volume and heat of adsorption on hydrogen uptake of IRMOF-1, IRMOF-4, IRMOF-6, IRMOF-7, IRMOF-8, IRMOF-10, IRMOF-12, IRMOF-14,IRMOF-16 and IRMOF-18 by carrying out GCMC simulations over a wide range of pressures at $77 \mathrm{~K}$. Their results showed that hydrogen adsorption is correlated with the heat of adsorption at low pressures ( $0.1 \mathrm{bar})$, with the surface area at intermediate pressures (30 bar) and with the free volume at high pressures (100 bar). Frost and Snurr ${ }^{38}$ later repeated the same correlation analysis at $298 \mathrm{~K}$ for IRMOF-1, IRMOF-9, IRMOF-10, IRMOF-14, IRMOF-16, and CuBTC and concluded that excess hydrogen adsorption is correlated well with the heat of adsorption at low pressures and with the surface area at the highest pressures.

Han and Goddard ${ }^{75}$ also used GCMC simulations to determine the contributions of heat of adsorption, surface area and pore volume to the excess and total hydrogen storage of MOFs, IRMOF-2-24, IRMOF-2-42, IRMOF-2-54, IRMOF-2-60, and IRMOF-2-96. They showed that at low pressures (1 bar), the excess hydrogen adsorption is determined mainly by the heat of adsorption, at intermediate pressures ( $30 \mathrm{bar}$ ), it is determined mostly by the free volume but also the surface area and at the highest pressure (100 bar) mostly by the surface area but also the free volume. These results were in perfect agreement with the findings of Frost et al. ${ }^{74}$ In the case of total hydrogen adsorption behavior, the order is similar to the excess adsorption with the exception that the order at 100 bar is the same as that at $30 \mathrm{bar}$, indicating that at $100 \mathrm{bar}$ the free volume of MOFs is the most important factor for hydrogen storage. Their results show that MOFs with high free volume are required to obtain the highest total hydrogen uptake, while MOFs with high surface area are required for the highest excess uptake. The excess hydrogen adsorption of these MOFs at 90 bar was found to correlate linearly with the calculated surface area. Here, it is important to discuss that at high pressures such as 90-100 bar, hydrogen adsorption may be due to simple occlusion, not due to the high isosteric heat of adsorption of the material. In other words, at these high pressures, MOFs with very large free volume have some advantages to qualify as good candidates for hydrogen storage, although they do not have high affinity for hydrogen. The free volume and surface area of MOFs can be increased by using large linkers; however, this is not going to be a real solution to achieve commercial 
hydrogen storage targets. It is also important to note that in these three studies, ${ }^{38,74,75}$ MOFs considered have the same topology and similar chemistry (IRMOFs); therefore, correlations were excellent and the transition between regimes occurred at the same pressures. However, there is a very large variety in the chemistry of MOFs and one should expect that the correlations may not be very good as the number and variety of materials considered increase.

Hydrogen adsorption in MOFs is a physisorption process and the interaction between hydrogen molecules and MOF atoms is very weak, resulting in low isosteric heat of adsorption. The molecular simulation studies summarized above indicated that it is a challenge to increase the isosteric heat of adsorption without significant losses in free volumes of MOFs. MOFs having smaller pores generally exhibit high heat of adsorption due to strong confinement of hydrogen molecules within the narrow pores. However, this type of material generally has lower free volume than MOFs having larger pores. Frost and Snurr ${ }^{38}$ showed that a new MOF with an isosteric heat of adsorption of 10-15 $\mathrm{kJ} \mathrm{mol}^{-1}$ and a free volume between 1.6 and $2.4 \mathrm{~cm}^{3} \mathrm{~g}^{-1}$ can achieve a hydrogen uptake of $6 \mathrm{wt} \%$, whereas MOFs with free volumes less than $1.5 \mathrm{~cm}^{3} \mathrm{~g}^{-1}$ will need heat of adsorption greater than $20 \mathrm{~kJ} \mathrm{~mol}^{-1}$ to achieve the same target. In order to increase the isosteric heat of adsorption, the interaction between hydrogen molecules and MOFs must be strengthened. Han and Goddard ${ }^{56}$ incorporated $\mathrm{Li}$ atoms in MOFs to strengthen the interaction between hydrogen molecules and framework. The GCMC simulations on Li-doped MOFs showed that hydrogen uptake of Li-MOF-C30 is 3.9 (4.6) wt\% at 20 (50) bar and $300 \mathrm{~K}$, which was the highest room temperature reversible hydrogen storage capacity that was reported at the time the study was conducted.

Bae and Snurr ${ }^{76}$ used GCMC simulations to demonstrate that the optimal isosteric heat of adsorption values for different MOFs (CuBTC, IRMOF-1, IRMOF-9, IRMOF-10, IRMOF-15, IRMOF-16, UMCM-1, MOF-177) are located in a very narrow range (18.5-22 $\mathrm{kJ} \mathrm{mol}^{-1}$ ) as Fig. 4 suggests. They concluded that increasing the heat of adsorption for MOFs having large surface areas $\left(>4800 \mathrm{~m}^{2} \mathrm{~g}^{-1}\right)$ is required to achieve the current hydrogen storage targets in terms of deliverable capacity. It is important to note that their study focused on deliverable capacity, the amount of hydrogen adsorbed at high pressure (120 bar) minus the amount adsorbed at the discharge pressure (1.5 bar). In contrast to most studies that focus on hydrogen uptake at high pressure, they considered the discharge of hydrogen from MOFs. They also concluded that a large surface area is more important than a large free volume to achieve the maximum delivery.

A systematic study was performed by Yang et al. ${ }^{77}$ to find out the effects of synthesis conditions on the evaluation of crystal structure, pore characteristics and hydrogen storage capacities of IRMOF-1 using experimental and computational tools. They showed that the synthesis method and activation procedure could be used to optimize the pore characteristics and hence the hydrogen uptake capacities of IRMOF-1 crystals. At 1 bar and $77 \mathrm{~K}$, interwoven IRMOF-1 crystals with high ultramicroporosity showed both high and fast hydrogen uptake, indicating that ultramicroporosity strongly affects the hydrogen uptake in MOFs at the low coverage. The smaller pore sizes or the higher ultramicropore volume leads to high heat of adsorption and better hydrogen uptake at low pressures. This work showed that focusing on the experimental synthesis conditions of MOFs may lead to design of materials with better uptake capacities.

Both experiments and simulations demonstrated that there is a linear relationship between the hydrogen storage capacity of MOFs at $77 \mathrm{~K}$ and the specific surface area of materials. ${ }^{4}$ Fig. 5 shows the relationship between hydrogen

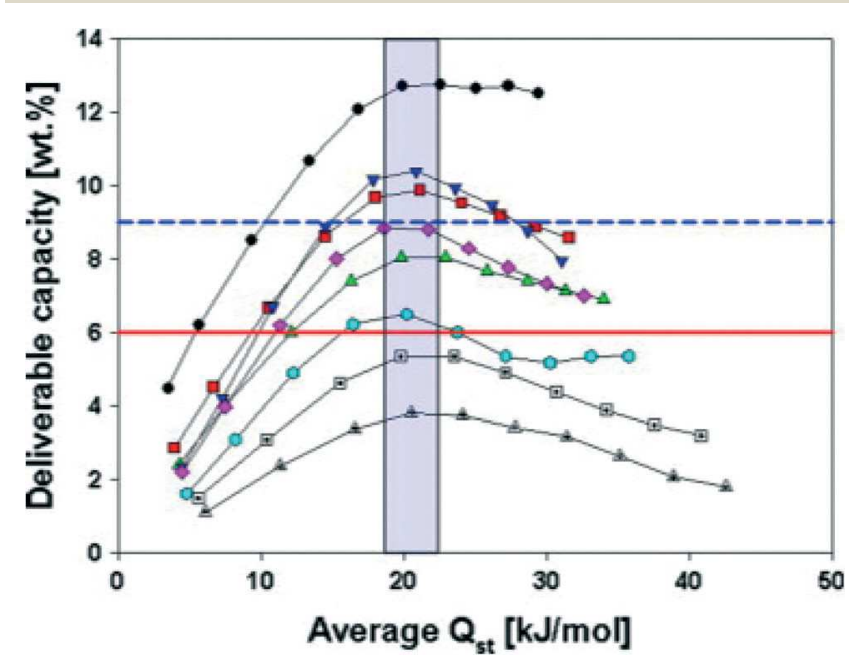

Fig. 4 Deliverable capacity from 120 to 1.5 bar vs. the average isosteric heat of adsorption for $\mathrm{H}_{2}$ adsorption at $298 \mathrm{~K}$ in the eight MOFs. Note that $Q_{\mathrm{st}}$ is averaged over the full pressure range (1.5-120 bar). The red (blue) line shows the 2010 (2015) DOE target. Reprinted with permission from ref. 76. Copyright (2010) Elsevier. MOFs from top to bottom: IRMOF-16, IRMOF-15, IRMOF-10, MOF-177, UMCM-1, IRMOF-1, IRMOF-9, CuBTC.

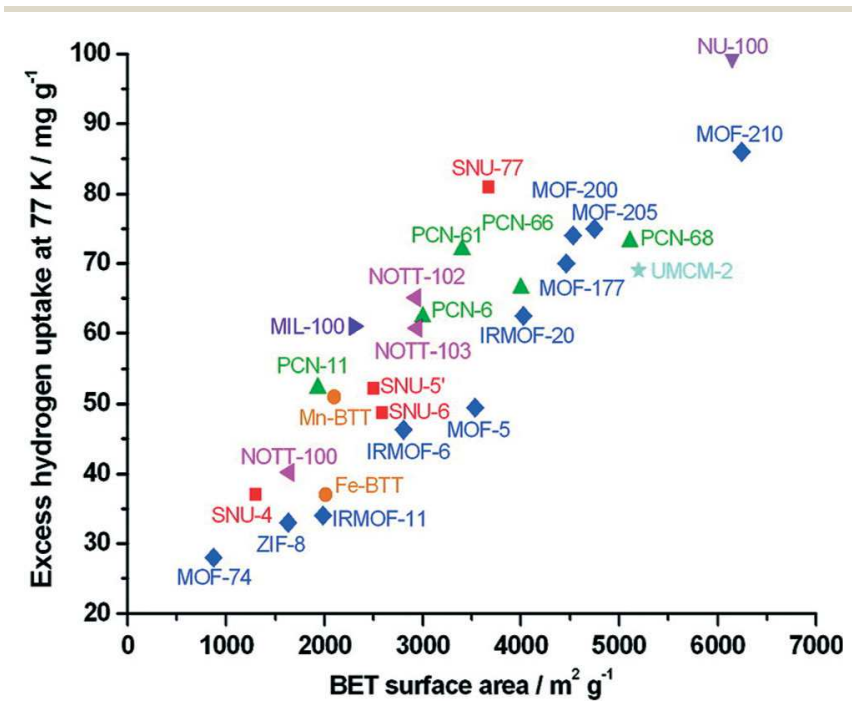

Fig. 5 Excess high-pressure hydrogen uptake capacities at $77 \mathrm{~K}$ versus BET surface areas for some highly porous MOFs. Reprinted with permission from ref. 4. Copyright (2012) American Chemical Society. 
uptake capacities of various MOFs at $77 \mathrm{~K}$ and high pressures as a function of surface areas of MOFs. At low pressures, around 1 bar, the correlation between uptake and surface area is not valid for materials having surface area $>2000 \mathrm{~m}^{2} \mathrm{~g}^{-1}$. Since pore volume is proportional to surface area, MOFs having both large surface area and high porosities are required to enhance hydrogen uptake capacities. Based on the results of these studies, it has been concluded that there are two critical structural parameters, surface area and free volume, which can be used to make predictions about the hydrogen uptake capacities of MOFs. Theoretical models that can make accurate predictions about the hydrogen storage potential of MOFs based on surface area and free volume are very efficient because they do not require the evaluation of interatomic potentials and computationally expensive isotherm simulations.

Kolotilov and Pavlishchuk ${ }^{78}$ found a linear relationship to predict hydrogen adsorption capacity of MOFs at $77 \mathrm{~K}$ and 1 bar using surface area and pore volume. They first tried to predict the adsorption capacity in weight percent based only on the surface area of MOFs and concluded that the equation based on the $S_{\text {Langmuir (Langmuir surface area) almost always }}$ makes higher predictions than the equation based on $S_{\text {BET }}$ (Brunauer, Emmett and Teller surface area). They then tried to evaluate the contributions of $S_{\mathrm{BET}}$ and pore volume for limiting sorption capacity ( $77 \mathrm{~K}$ and 1 bar) of MOFs by using the same equation that they have derived for silica molecular sieves. In this equation $N$ is the adsorption of hydrogen in $\mathrm{wt} \%, a$ is the mass of the hydrogen adsorbed on $1 \mathrm{~m}^{2}$ of surface in $\mathrm{g} \times 100, S_{\mathrm{BET}}$ is the BET surface area in $\mathrm{m}^{2} \mathrm{~g}^{-1}, b$ is the density of hydrogen in the micropores in $\mathrm{g} \mathrm{cm}^{-3} \times 100$ and $V_{\text {pore }}$ is the pore volume in $\mathrm{cm}^{3} \mathrm{~g}^{-1}$ :

$$
N=a \times S_{\mathrm{BET}}+b \times V_{\text {pore }}
$$

The coefficients $a$ and $b$ were derived from the simplex method by minimizing the error between experimental measurements and predictions of equation. The best fit for MOFs was obtained with $a=2.1 \times 10^{-3}\left( \pm 0.1 \times 10^{-3}\right)\left(\mathrm{H}_{2} \mathrm{wt} \%\right)$ and $b=0.1( \pm 0.02)\left(\mathrm{H}_{2} \mathrm{wt} \%\right)$. Even though the physical meaning of the coefficient $b$ is the density of hydrogen attainable in microporous sorbents, the value used in this equation is considerably lower. The density of the hydrogen at $77 \mathrm{~K}$ and 1 bar is $0.04 \mathrm{~g} \mathrm{~cm}^{-3}$ but the value used for $b$ is $0.001 \mathrm{~g} \mathrm{~cm}^{-3}$. This was attributed to the fact that the pore volume in the equation represents the pores in which hydrogen does not condense. ${ }^{78}$ Using eqn (1), the hydrogen adsorption capacity (wt\%) of MOFs at $77 \mathrm{~K}$ and 1 bar can be easily predicted because both BET surface area and pore volume are easily computable using the method developed by Düren et al. ${ }^{79}$ and PLATON software. ${ }^{80}$

Goldsmith et $a .^{81}$ have recently worked on the same equation to estimate the maximum theoretical hydrogen uptake (at $77 \mathrm{~K}$ and 35 bar) of MOFs. To obtain the maximum theoretical uptake, a material should be free of all solvent molecules and pore volume should be calculated to be the micropore volume within a single crystal monolith of the MOF. With these assumptions, they estimated the total amount of hydrogen adsorbed by a specific MOF ( $\left.n_{\text {Total }}\right)$ using the following equation:

$$
n_{\text {Total }}=n_{\text {Excess }}+n_{\text {gas }}=C \times S+\rho_{\mathrm{H}_{2}} \times V_{\text {Pore }}
$$

In this equation $\rho_{\mathbf{H}_{2}}$ is the density of hydrogen at $77 \mathrm{~K}$ and 35 bar $\left(11.5 \mathrm{~kg} \mathrm{~m}^{-3}\right), V_{\text {Pore }}$ is the pore volume that they calculated using PLATON software, ${ }^{80} S$ is the surface area calculated using the algorithm by Düren et al. ${ }^{79} C$ is the proportionality constant $\left(0.021 \mathrm{mg} \mathrm{H}_{2} \mathrm{~m}^{-2}\right)$ only specific at $77 \mathrm{~K}$ and 35 bar. If $n_{\text {Total }}$ is divided by the mass of the unit cell, total gravimetric uptake of hydrogen ( $\mathrm{g}$ hydrogen per $\mathrm{g}$ MOF) is obtained.

Goldsmith et al. ${ }^{81}$ screened more than 600000 entries from the Cambridge Structural Database (CSD) and ended up with more than 4000 solvent-free MOF structures to identify the most promising ones for hydrogen storage. They concluded that SNU-21 is the best candidate with $12.4 \mathrm{wt} \%$ and $71.4 \mathrm{~g} \mathrm{~L}^{-1}$ hydrogen storage capacity. However, experiments ${ }^{82}$ did not achieve this high performance which was attributed to the incomplete solvent removal from the MOF during synthesis. Several other MOFs were identified to pass the DOE targets with 10-11.4 wt\% and $\sim 60 \mathrm{~g} \mathrm{~L} \mathrm{~L}^{-1}$ hydrogen uptakes but these MOFs have not been tested by experiments yet. That study by Goldsmith et $a l .{ }^{81}$ also showed that MOF density decreases with increasing surface area, indicating that there is a trade-off between gravimetric and volumetric hydrogen storage capacity of MOFs. Fig. 6 shows that volumetric and gravimetric densities are positively correlated at capacities below $\sim 5 \mathrm{wt} \%$, but at higher wt $\%$ the volumetric density approaches a maximum of $\sim 64 \mathrm{~g} \mathrm{~L}^{-1}$ and then decreases for MOFs having larger surface areas. That means MOFs having large surface areas $\left(>\sim 5500 \mathrm{~m}^{2} \mathrm{~g}^{-1}\right)$ cannot meet the DOE volumetric target and the most promising materials have moderate surface areas (3100-4800 $\mathrm{m}^{2} \mathrm{~g}^{-1}$ ) resulting in both high gravimetric values of $8-12 \mathrm{wt} \%$ and volumetric densities of 50-64 $\mathrm{g} \mathrm{L}^{-1}$. These results suggested that MOFs must have moderate mass densities $\left(>0.5 \mathrm{~g} \mathrm{~cm}^{-3}\right)$ with high surface areas in order to meet the desired hydrogen storage targets.

Gomez and Sastre ${ }^{83}$ tried to establish a range of desired MOF densities to meet the gravimetric and volumetric target. They showed that under reasonable conditions both cannot be achieved with MOFs whose density is lower than $0.7 \mathrm{~g} \mathrm{~cm}^{-3}$ because reaching the ultimate hydrogen volumetric uptake $\left(70 \mathrm{~g} \mathrm{~L}^{-1}\right)$ would require gravimetric uptakes larger than $10 \%$, which is unlikely in MOFs. Instead, if the MOF density is $1.0 \mathrm{~g} \mathrm{~cm}^{-3}$, such a volumetric target can be reached with a gravimetric uptake lower than $8 \%$, still high for current results in MOFs. Gomez et al. ${ }^{84}$ have very recently screened a database containing approximately 138000 MOFs to select candidate structures for hydrogen storage. A total of 231 structures (of which 79 contain paddle-wheel units) were selected that meet the gravimetric and volumetric targets at 


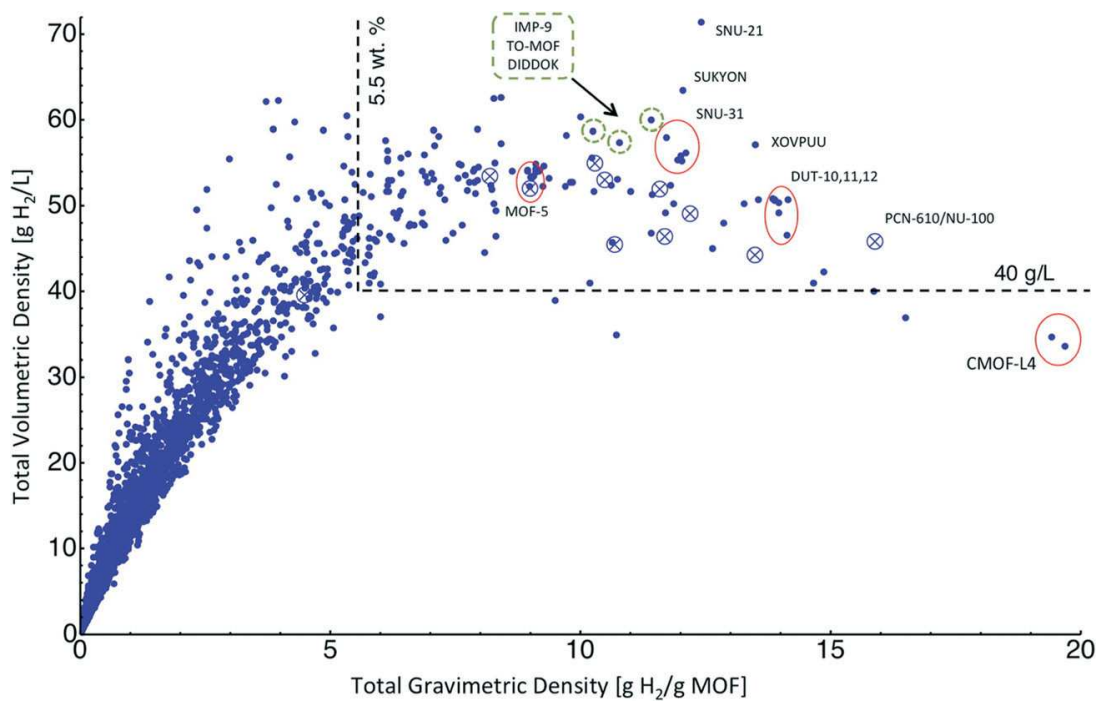

Fig. 6 Theoretical total (adsorbed + gas phase $\mathrm{H}_{2}$ at $77 \mathrm{~K}$ and 35 bar) volumetric and gravimetric density of stored $\mathrm{H}_{2}$ in $\sim 4000 \mathrm{MOFs}$ mined from the Cambridge Structural Database (CSD). The data account only for the mass and volume of the MOF media; mass and volume contributions from the system are neglected. For comparison, the region bounded by the dashed lines represents the DOE 2017 targets for $\mathrm{H}_{2}$ storage systems. Crossed circles represent common MOFs with incomplete or disordered crystal data in the CSD; structures for these compounds were constructed by hand. Reprinted with permission from ref. 81. Copyright (2013) American Chemical Society.

$100 \mathrm{~atm}$ and $77 \mathrm{~K}$. They used GCMC simulations to calculate the hydrogen adsorption isotherms and select structures which meet the targets at $50 \mathrm{~atm}$. From this a reduced set of 18 structures were analyzed regarding gravimetric and volumetric uptake and pore size distribution and pore volume. A few structures with $3 \%$ gravimetric uptake at 1 atm and $77 \mathrm{~K}$ perform at the best level found so far. Their results showed that several hypothetical structures give uptake similar to those of IRMOF-62, and HKUST-1 (and its isostructural Zn derivative) is the best paddle-wheel-based structure for hydrogen adsorption. They concluded that these results are still far from the commercial targets and applying similar analyses to larger databases and other topologies would help in selecting new subfamilies of MOFs which could be better candidates to approach the commercial targets required.

Since all these studies have focused on the effects of surface area and porosity of MOFs on their hydrogen storage, experimental studies are directed to increase these two properties. Several methods have been suggested to increase the surface area of MOFs such as using polycarboxylate ligands as organic building blocks, elongation of the ligands, and employing mixed ligands in the synthesis of MOFs. Suh et al. ${ }^{4}$ discussed that MOFs constructed from hexacarboxylic acid have higher surface areas and pore volumes compared to ones having di-, tri-, or tetracarboxylates. Therefore, MOFs with hexacarboxylic acid have higher hydrogen storage capacities compared to materials with other organic linkers.

Finally, it is important to note that gas uptake properties of nanoporous materials are determined by several criteria and it is somehow a simplification to think that hydrogen storage of MOFs is determined by only two structural properties (surface area and pore volume). Other chemical and physical properties such as pore sizes and shapes, crystal densities, chemical topology, type of the organic linkers and metals, availability of the binding sites, purity of the materials, etc. may all have significant effects on the gas uptake capacity of MOFs. Surface area and porosity have been widely studied perhaps due to the availability of well-known and validated methods that quantitatively characterize these two properties. More extensive models that can take other chemical and physical properties into account will be much more useful for further assessment of hydrogen storage in MOFs. It must be also noted that the two equations listed above make predictions for limiting ( 1 bar) and maximum ( 35 bar) hydrogen uptake of MOFs at $77 \mathrm{~K}$. Models that can predict hydrogen uptake as a function of pressure and temperature would be extremely useful to assess the potential of MOFs.

\section{Outlook}

We summarized the recent advances in using molecular simulations and theoretical models to predict the hydrogen adsorption capacities of MOFs. Of course, the combination of simulation methods with the experiments is a must to accurately evaluate the potential of a MOF material and to design new MOFs that will exhibit high performance in hydrogen storage. Considering the very large number of available MOF materials, it would be better to systematically screen MOFs using molecular simulations to identify the most promising materials for hydrogen storage. Experimental efforts, resources and time can then be directed to these highly promising materials.

Molecular simulations and theoretical models can make accurate estimates about the MOFs' hydrogen uptake 
capacities but experiments must be performed to evaluate the practical usage of MOFs. For example, many MOFs are known to degrade under the presence of a humid environment by losing their crystallographic structure. ${ }^{85}$ It has been discussed that MOFs having open metal sites are promising for hydrogen storage due to the strong interaction between hydrogen molecules and metal atoms. ${ }^{86-90}$ Space et al. ${ }^{91,92}$ simulated the hydrogen adsorption mechanism in MOFs with open metal sites. Fisher et al. ${ }^{93}$ reviewed the recent developments in the application of quantum mechanical methods and classical molecular simulations to understand and predict adsorption in MOFs with coordinatively unsaturated metal sites. It has been demonstrated how accurate potential parameters for the interaction of hydrogen with unsaturated metal sites can be derived from Density Functional Theory calculations for a simple model system. ${ }^{94}$ On the other hand, exposure of some MOFs to humidity may result in coordination of water molecules with the open metal site and reduce the expected hydrogen uptake. This type of MOF will not be useful for hydrogen storage even though simulations and/or theoretical models identify it with a high storage capacity. The GCMC simulations that we discussed in this review are not able to make predictions about the long-term stability of MOFs and perhaps this is the most open area for further research in this field. Simulations predicting the stability of MOFs under the presence of moisture, air, and other gas impurities simultaneously with their hydrogen storage potential will have a large impact.

Since the accuracy of molecular simulations is mainly determined by the force fields and potential models used to describe hydrogen-hydrogen and hydrogen-MOF interactions, development of reliable force fields from the quantum-level calculations is important and open for research. Development of force fields for flexible MOFs and that can predict the hydrogen uptake and structural change in the framework at the same time will be very useful. Similarly, development of new force fields from quantum mechanical calculations is needed to describe the strong interactions between hydrogen molecules and open metal sites of MOFs.

Results of molecular simulations and theoretical models discussed in this review indicate that most of the as-synthesized MOFs are not able to achieve the DOE targets for hydrogen storage but they can satisfy the targets even at near-ambient temperature after functionalization and/or chemical modification of the framework. With the current speed of advancement in computers, molecular simulations will be much more useful in the future by providing atomic-level information about the specific hydrogen-MOF interactions. This information will be helpful to identify MOFs having high hydrogen storage potential before carrying out extensive experiments in addition to leading experiments for design and development of new MOFs with extremely good hydrogen storage capacities.

\section{Acknowledgements}

S. K. acknowledges and thanks the TUBA-GEBIP Programme.

\section{References}

1 L. Schlapbach and A. Zuttel, Nature, 2001, 414, 353.

2 http://energy.gov/eere/fuelcells/hydrogen-storage-currenttechnology.

3 H. W. Langmi and G. S. McGrady, Coord. Chem. Rev., 2007, 251, 925.

4 M. P. Suh, H. J. Park, T. K. Prasad and D. W. Lim, Chem. Rev., 2012, 112, 782.

5 B. J. Burnett, P. M. Barron and W. Choe, CrystEngComm, 2012, 14, 3839.

6 O. M. Yaghi and H. L. Li, J. Am. Chem. Soc., 1995, 117, 10401.

7 M. Eddaoudi, H. L. Li, T. Reineke, M. Fehr, D. Kelley, T. L. Groy and O. M. Yaghi, Top. Catal., 1999, 9, 105.

8 H. Li, M. Eddaoudi, M. O'Keeffe and O. M. Yaghi, Nature, 1999, 402, 276.

9 O. M. Yaghi, M. O'Keeffe, N. W. Ockwig, H. K. Chae, M. Eddaoudi and J. Kim, Nature, 2003, 423, 705.

10 K. Schlichte, T. Kratzke and S. Kaskel, Microporous Mesoporous Mater., 2004, 73, 81.

11 A. C. Sudik, A. R. Millward, N. W. Ockwig, A. P. Cote, J. Kim and O. M. Yaghi, J. Am. Chem. Soc., 2005, 127, 7110.

12 A. Pichon, A. Lazuen-Garay and S. L. James, CrystEngComm, 2006, 8, 211.

13 A. P. Cote and O. M. Yaghi, Abstr. Pap. Am. Chem. Soc., 2007, 233, 61.

14 H. M. El-Kaderi, J. R. Hunt, J. L. Mendoza-Cortés, A. P. Côté, R. E. Taylor, M. O'Keeffe and O. M. Yaghi, Science, 2007, 316, 268.

15 R. Banerjee, A. Phan, B. Wang, C. Knobler, H. Furukawa, M. O'Keeffe and O. M. Yaghi, Science, 2008, 319, 939.

16 A. Phan, C. J. Doonan, F. J. Uribe-Romo, C. B. Knobler, M. O'Keeffe and O. M. Yaghi, Acc. Chem. Res., 2009, 43, 58.

17 P. J. Beldon, L. Fabian, R. S. Stein, A. Thirumurugan, A. K. Cheetham and T. Friscic, Angew. Chem., Int. Ed., 2010, 49, 9640.

18 Q. R. Fang, T. A. Makal, M. D. Young and H. C. Zhou, Comments Inorg. Chem., 2010, 31, 165.

19 L. Hertäg, H. Bux, J. Caro, C. Chmelik, T. Remsungnen, M. Knauth and S. Fritzsche, J. Membr. Sci., 2011, 377, 36.

20 Y. B. Sun, L. Wang, W. A. Amer, H. J. Yu, J. Ji, L. Huang, J. Shan and R. B. Tong, J. Inorg. Organomet. Polym. Mater., 2013, 23, 270.

21 S. T. Meek, J. A. Greathouse and M. D. Allendorf, $A d v$. Mater., 2011, 23, 249.

22 M. Eddaoudi, J. Kim, N. Rosi, D. Vodak, J. Wachter, M. O'Keeffe and O. M. Yaghi, Science, 2002, 295, 469.

23 S. Keskin and S. Kizilel, Ind. Eng. Chem. Res., 2011, 50, 1799.

24 A. R. Millward and O. M. Yaghi, J. Am. Chem. Soc., 2005, 127, 17998.

25 U. Mueller, M. Schubert, F. Teich, H. Puetter, K. Schierle-Arndt and J. Pastré, J. Mater. Chem., 2006, 16, 626.

26 L. Pan, M. B. Sander, X. Y. Huang, J. Li, M. Smith, E. Bittner, B. Bockrath and J. K. Johnson, J. Am. Chem. Soc., 2004, 126, 1308. 
27 Y. G. Zhao, H. H. Wu, T. J. Emge, Q. H. Gong, N. Nijem, Y. J. Chabal, L. Z. Kong, D. C. Langreth, H. Liu, H. P. Zeng and J. Li, Chem. - Eur. J., 2011, 17, 5101.

28 A. R. Millward and O. M. Yaghi, J. Am. Chem. Soc., 2005, 127, 17998.

29 M. Eddaoudi, H. L. Li and O. M. Yaghi, J. Am. Chem. Soc., 2000, 122, 1391.

30 Z. H. Xiang, D. P. Cao, J. H. Lan, W. C. Wang and D. P. Broom, Energy Environ. Sci., 2010, 3, 1469.

31 J. W. Jiang, R. Babarao and Z. Q. Hu, Chem. Soc. Rev., 2011, 40, 3599.

32 R. B. Getman, Y. S. Bae, C. E. Wilmer and R. Q. Snurr, Chem. Rev., 2012, 112, 703.

33 J. Xiao, M. Hu, P. Bénard and R. Chahine, Int. J. Hydrogen Energy, 2013, 38, 13000.

34 S. S. Han, J. L. Mendoza-Cortes and W. A. Goddard, Chem. Soc. Rev., 2009, 38, 1460.

35 D. Frenkel and B. Smit, Understanding Molecular Simulation: From Algorithms to Applications, Academic Press, San Diego, 2nd edn, 2002.

36 A. L. Myers and P. A. Monson, Langmuir, 2002, 18, 10261.

37 H. Furukawa, M. A. Miller and O. M. Yaghi, J. Mater. Chem., $2007,17,3197$.

38 H. Frost and R. Q. Snurr, J. Phys. Chem. C, 2007, 111, 18794.

39 A. K. Rappe, C. J. Casewit, K. S. Colwell, W. A. Goddard and W. M. Skiff, J. Am. Chem. Soc., 1992, 114, 10024.

40 S. L. Mayo, B. D. Olafson and W. A. Goddard, J. Phys. Chem., 1990, 94, 8897.

41 W. L. Jorgensen, D. S. Maxwell and J. TiradoRives, J. Am. Chem. Soc., 1996, 118, 11225.

42 J. E. Lennard-Jones, Proc. Phys. Soc., London, 1931, 43, 461.

43 V. Buch, J. Chem. Phys., 1994, 100, 7610.

44 Q. U. Wang, J. K. Johnson and J. Q. Broughton, Mol. Phys., 1996, 89, 1105.

45 F. Darkrim and D. Levesque, J. Chem. Phys., 1998, 109, 4981.

46 R. P. Feynman and A. R. Hibbs, Quantum Mechanics and Path Integrals, McGraw-Hill, New York, 1965.

47 J. C. Liu, R. B. Rankin and J. K. Johnson, Mol. Simul., 2009, 35, 60 .

48 Q. Y. Yang and C. L. Zhong, J. Phys. Chem. B, 2006, 110, 655.

49 G. Garberoglio, A. I. Skoulidas and J. K. Johnson, J. Phys. Chem. B, 2005, 109, 13094.

50 Q. Yang and C. Zhong, J. Phys. Chem. B, 2005, 109, 11862.

51 S. S. Han, W. Q. Deng and W. A. Goddard, Angew. Chem., Int. Ed., 2007, 46, 6289.

52 R. B. Getman, J. H. Miller, K. Wang and R. Q. Snurr, J. Phys. Chem. C, 2011, 115, 2066.

53 L. Zhang, Q. Wang and Y. C. Liu, J. Phys. Chem. B, 2007, 111, 4291.

54 D. H. Jung, D. Kim, T. B. Lee, S. B. Choi, J. H. Yoon, J. Kim, K. Choi and S. H. Choi, J. Phys. Chem. B, 2006, 110, 22987.

55 P. Ryan, L. J. Broadbelt and R. Q. Snurr, Chem. Commun., 2008, 4132.
56 S. S. Han and W. A. Goddard, J. Am. Chem. Soc., 2007, 129, 8422.

57 D. P. Cao, J. H. Lan, W. C. Wang and B. Smit, Angew. Chem., Int. Ed., 2009, 48, 4730.

58 R. Babarao, M. Eddaoudi and J. W. Jiang, Langmuir, 2010, 26, 11196.

59 O. K. Farha, A. O. Yazaydin, I. Eryazici, C. D. Malliakas, B. G. Hauser, M. G. Kanatzidis, S. T. Nguyen, R. Q. Snurr and J. T. Hupp, Nat. Chem., 2010, 2, 944.

60 Z. H. Xiang, D. P. Cao, J. H. Lan, W. C. Wang and D. P. Broom, Energy Environ. Sci., 2010, 3, 1469.

61 J. Sculley, D. Q. Yuan and H. C. Zhou, Energy Environ. Sci., 2011, 4, 2721.

62 R. Babarao, S. Dai and D. E. Jiang, J. Phys. Chem. C, 2011, 115, 8126.

63 M. P. Suh, Y. E. Cheon and E. Y. Lee, Chem. - Eur. J., 2007, 13, 4208.

64 T. Loiseau, C. Serre, C. Huguenard, G. Fink, F. Taulelle, M. Henry, T. Bataille and G. Ferey, Chem. - Eur. J., 2004, 10, 1373.

65 N. Nijem, P. Thissen, Y. P. Yao, R. C. Longo, K. Roodenko, H. H. Wu, Y. G. Zhao, K. Cho, J. Li, D. C. Langreth and Y. J. Chabal, J. Am. Chem. Soc., 2011, 133, 12849.

66 F. X. Coudert, M. Jeffroy, A. H. Fuchs, A. Boutin and C. Mellot-Draznieks, J. Am. Chem. Soc., 2008, 130, 14294.

67 M. J. Rosseinsky, Microporous Mesoporous Mater., 2004, 73, 15. 68 X. J. Wu, X. Yang, J. Song and W. Q. Cai, Chin. J. Chem., 2012, 70, 2518.

69 E. Pantatosaki, H. Jobic, D. I. Kolokolov, S. Karmakar, R. Biniwale and G. K. Papadopoulos, J. Chem. Phys., 2013, 138, 034706.

70 Q. Shi, Z. W. Song, X. Z. Kang, J. X. Dong and Y. Zhang, CrystEngComm, 2012, 14, 8280.

71 D. J. Zhang, T. Y. Song, J. Shi, K. R. Ma, Y. Wang, L. Wang, P. Zhang, Y. Fan and J. N. Xu, Inorg. Chem. Commun., 2008, 11, 192.

72 J. C. Liu, J. T. Culp, S. Natesakhawat, B. C. Bockrath, B. Zande, S. G. Sankar, G. Garberoglio and J. K. Johnson, J. Phys. Chem. C, 2007, 111, 9305.

73 S. Keskin, J. Liu, R. B. Rankin, J. K. Johnson and D. S. Sholl, Ind. Eng. Chem. Res., 2009, 48, 2355.

74 H. Frost, T. Duren and R. Q. Snurr, J. Phys. Chem. B, 2006, 110, 9565.

75 S. S. Han and A. W. Goddard III, J. Phys. Chem. C, 2008, 112, 13431.

76 Y. S. Bae and R. Q. Snurr, Microporous Mesoporous Mater., 2010, 132, 300.

77 S. J. Yang, J. H. Cho, K. Lee, T. Kim and C. R. Park, Chem. Mater., 2010, 22, 6138.

78 S. V. Kolotilov and V. V. Pavlishchuk, Theor. Exp. Chem., 2009, 45, 75 .

79 T. Düren, F. Millange, G. Ferey, K. S. Walton and R. Q. Snurr, J. Phys. Chem. C, 2007, 111, 15350.

80 P. A. L. Spek, A Multipurpose Crystallographic Tool, Utrecht University, 2010.

81 J. Goldsmith, A. G. Wong-Foy, M. J. Cafarella and D. J. Siegel, Chem. Mater., 2013, 25, 3373.

82 T. K. Kim and M. P. Suh, Chem. Commun., 2011, 47, 4258. 
83 D. A. Gomez and G. Sastre, Phys. Chem. Chem. Phys., 2011, 13, 16558.

84 D. A. Gomez, J. Toda and G. Sastre, Phys. Chem. Chem. Phys., 2014, 16, 19001.

85 J. A. Greathouse and M. D. Allendorf, J. Am. Chem. Soc., 2006, 128, 10678.

86 A. Uzun and S. Keskin, Prog. Surf. Sci., 2014, 89, 56.

87 P. D. C. Dietzel, B. Panella, M. Hirscher, R. Blom and H. Fjellvag, Chem. Commun., 2006, 959.

88 B. L. Chen, N. W. Ockwig, A. R. Millward, D. S. Contreras and O. M. Yaghi, Angew. Chem., Int. Ed., 2005, 44, 4745.

89 W. Zhou, H. Wu and T. Yildirim, J. Am. Chem. Soc., 2008, 130, 15268.

90 K. Sumida, C. M. Brown, Z. R. Herm, S. Chavan, S. Bordiga and J. R. Long, Chem. Commun., 2011, 47, 1157.

91 K. A. Forrest, T. Pham, K. McLaughlin, J. L. Belof, A. C. Stern, M. J. Zaworotko and B. Space, J. Phys. Chem. C, 2012, 116, 15538.

92 T. Pham, K. A. Forrest, P. Nugent, Y. Belmabkhout, R. Luebke, M. Eddaoudi, M. J. Zaworotko and B. Space, J. Phys. Chem. C, 2013, 117, 9340.

93 M. Fischer, J. R. B. Gomes and M. Jorge, Mol. Simul., 2014, 40, 537.

94 M. Fischer, B. Kuchta, L. Firlej, F. Hoffman and M. Froba, J. Phys. Chem. C, 2010, 114, 19116.

95 M. Gallo and D. Glossman-Mitnik, J. Phys. Chem. C, 2009, 113, 6634.

96 J. Liu, J. Y. Lee, L. Pan, R. T. Obermyer, S. Simizu, B. Zande, J. Li, S. G. Sankar and J. K. Johnson, J. Phys. Chem. C, 2008, 112, 2911.
97 R. B. Rankin, J. C. Liu, A. D. Kulkarni and J. K. Johnson, J. Phys. Chem. C, 2009, 113, 16906.

98 J. W. Jiang, AIChE J., 2009, 55, 2422.

99 T. Sagara, J. Klassen and E. Ganz, J. Chem. Phys., 2004, 121, 12543.

100 S. S. Han, H. Furukawa, O. M. Yaghi and W. A. Goddard, J. Am. Chem. Soc., 2008, 130, 11580.

101 E. Klontzas, E. Tylianakis and G. E. Froudakis, J. Phys. Chem. C, 2008, 112, 9095.

102 Z. H. Xiang, J. H. Lan, D. P. Cao, X. H. Shao, W. C. Wang and D. P. Broom, J. Phys. Chem. C, 2009, 113, 15106.

103 D. Noguchi, H. Tanaka, A. Kondo, H. Kajiro, H. Noguchi, T. Ohba, H. Kanoh and K. Kaneko, J. Am. Chem. Soc., 2008, 130, 6367.

104 M. Fischer, F. Hoffmann and M. Froba, ChemPhysChem, 2009, 10, 2647.

105 E. Atci, I. Erucar and S. Keskin, J. Phys. Chem. C, 2011, 115, 6833.

106 I. Erucar and S. Keskin, Ind. Eng. Chem. Res., 2013, 52, 3462.

107 S. Keskin, J. Phys. Chem. C, 2011, 116, 1772.

108 S. Keskin, Mol. Simul., 2013, 39, 14.

109 T. N. Ozturk and S. Keskin, Ind. Eng. Chem. Res., 2013, 52, 17627.

110 A. G. Wong-Foy, A. J. Matzger and O. M. Yaghi, J. Am. Chem. Soc., 2006, 128, 3494.

111 B. Panella, M. Hirscher, H. Putter and U. Muller, Adv. Funct. Mater., 2006, 16, 520.

112 A. Dailly, J. J. Vajo and C. C. Ahn, J. Phys. Chem. B, 2006, 110, 1099. 